\title{
Psicólogos escolares em Portugal: perfil e necessidades de formação
}

\author{
School psychologists in Portugal: Training \\ profile and educational needs
}

\author{
Sofia Abreu MENDES \\ Isabel ABREU-LIMA 1 \\ Leandro Silva ALMEIDA²
}

\begin{abstract}
Resumo
Tendo por base um inquérito junto a 477 psicólogos escolares de instituições públicas e privadas portuguesas, foram analisados o perfil e as necessidades de formação desse grupo profissional. Os resultados apontam que, a par da formação em áreas diretamente relacionadas com a prática psicológica em contexto escolar, muitos profissionais reportaram ser especializados no domínio da psicologia clínica e da saúde. As necessidades de formação identificadas parecem refletir a natureza dos problemas encontrados na área, como as dificuldades de aprendizagem, a indisciplina, e os problemas de comportamento. Tais necessidades parecem ainda apontar as principais áreas de intervenção desses profissionais, atinentes a necessidades educativas especiais, avaliação e consulta psicológica, e orientação vocacional. Paralelamente, elas evidenciam o desejo desses profissionais em expandir seu campo de atuação para além dos papéis ditos tradicionais. A partir deste estudo podem ser derivadas implicações tanto para a formação inicial quanto para a formação continuada dos psicólogos escolares.
\end{abstract}

Palavras-chave: Educação; Educação continuada; Portugal; Psicologia escolar; Psicólogos.

\begin{abstract}
The profile and training needs of Portuguese school psychologists, working in public and private schools, were analysed based on a survey with 477 professionals. Results show that in conjunction with training in domains directly related to psychological practice in the school context, many school psychologists reported having a specialization course in the field of clinic and/or health psychology. The identified training needs appear to reflect the nature of the problems encountered in the field (e.g., learning difficulties, indiscipline and behaviour problems), as well as the main intervention areas of Portuguese school psychologists (e.g., special educational needs, assessment, support/counselling and vocational guidance). In parallel, results show that some professionals desire to expand their field of expertise beyond the so-called traditional roles. We discuss the implications for the initial and continuing training of school psychologists.

Keywords: Education; Continuing education; Portugal; School psychology; Psychologists.

$\operatorname{Mrv}$

1 Universidade do Porto, Faculdade de Psicologia e de Ciências de Educação. R. Alfredo Allen, 4200-135, Porto, Portugal. Correspondência para/Correspondence to: S.A. MENDES. E-mail: <sofia.a.mendes@gmail.com>.

2 Universidade do Minho, Instituto de Educação. Braga, Portugal.

Apoio: Fundação para a Ciência e Tecnologia (Bolsa de doutoramento SFRH/BD/78646/2011).
\end{abstract}


A Psicologia Escolar sofreu nas últimas décadas um crescimento significativo enquanto área de conhecimento e profissão. Presentemente, existem evidências do campo em pelo menos 83 países dos vários continentes, observando-se uma grande variabilidade no grau de estabelecimento e na forma como essa disciplina se configura nos diferentes cenários educativos (Jimerson, Skokut, Cadernas, Malone, \& Stewart, 2008). Para tal diversidade contribuem fatores socioculturais, políticos e econômicos, mas também a fase de desenvolvimento em que se encontra a Educação e a Psicologia em cada país (Oakland \& Jimerson, 2007).

Em Portugal, a intervenção psicológica no ensino não superior tem como enquadramento os Serviços de Psicologia e Orientação (SPO). Esses serviços são objeto de regulamentação própria desde o início da década de 1990 (Decreto-Lei n 190/91), a qual, para além de ter formalizado essas estruturas dentro do sistema escolar, definiu atribuições, moldes de funcionamento e organização. Na linha desse decreto-lei, a ação dos SPO abrange diversos públicos-alvo (alunos, família, escola e comunidade), diversas áreas (apoio psicopedagógico, apoio ao desenvolvimento do sistema de relações da comunidade educativa, orientação escolar e vocacional), e diferentes níveis de intervenção (remediativo, preventivo e promocional). Tem em vista a promoção da igualdade de oportunidades, o sucesso escolar e a aproximação entre a família, a escola e o mundo das atividades profissionais (Almeida, 2003).

No panorama nacional, a análise da prática psicológica em contexto escolar tem sido sobretudo relegada ao plano teórico, sendo menos frequente a produção de caráter empírico ou com amostras representativas desses profissionais (Mendes, Abreu-Lima, \& Almeida, 2013a; Mendes, Abreu-Lima, Almeida, \& Simeonsson, 2014). Os estudos realizados até à presente data apontam para uma concentração dos SPO nos papéis ditos tradicionais, em intervenções de caráter remediativo, bem como uma atuação centrada no aluno. Isso parece ser fruto de expectativas inadequadas quanto aos serviços, bem como do crescente número de alunos sinalizados com necessidades de apoio e, ainda, da pectos (Centro Interdisciplinar de Estudos Econômicos [CIDEC], 2006; Leitão, Paixão, Silva, \& Miguel, 2001; Mendes et al., 2014; Ministério da Educação, 2000)

Esse padrão de atividade, não sendo exclusivo dos psicólogos escolares portugueses, tem sido amplamente criticado por ser questionável em termos de eficácia, e por contrariar o modelo de intervenção desejável para o contexto escolar. Este propõe para o psicólogo uma ação predominantemente preventiva e promocional nos seus objetivos, e ecológico-sistêmica na sua concepção (Almeida, 2003; Almeida, Franco, Soares, Alves, \& Gonçalves, 2013; Almeida \& Peixoto, 2011; Barbosa \& Marinho-Araújo, 2010; Farrel, 2010; Gutkin, 2012). A despeito desse criticismo, os estudos disponíveis evidenciam uma avaliação globalmente positiva dos SPO por parte da comunidade educativa, sugerindo a pertinência e a valorização do trabalho desenvolvido (CIDEC, 2006; Leitão et al., 2001; Ministério da Educação, 2000).

A par do enquadramento legal que fixa os parâmetros e orientações para o exercício da profissão, a atuação dos psicólogos escolares é ainda influenciada por um conjunto heterogêneo de fatores, de entre os quais se destaca a sua própria formação (Fagan \& Wise, 2007). Organizações profissionais descrevem o psicólogo escolar como um profissional especializado na área da Psicologia e da Educação (European Federation of Psychologists' Associations [EFPA], 2010; National Association of School Psychologists [NASP], 2010; Oakland \& Cunningham, 1992), definindo inclusivamente o perfil das competências teórico-práticas desejáveis.

Para além de reconhecida como crucial para uma atuação competente diante dos desafios do sistema educativo (Guzzo, Almeida, \& Wechsler, 2001; Marinho-Araújo \& Neves, 2007), a necessidade de uma formação especializada é ainda identificada como crítica para a consolidação da identidade do psicólogo escolar (Marinho-Araújo, 2005a; 2005b). Não obstante, intra e inter países, observase uma grande diversidade nos programas de educação e treino em Psicologia Escolar, principalmente quanto aos critérios de acesso, duração, natureza e tempo dos estágios, e na habilitação para o exercício da profissão (e.g., bacharelado, 
especialização, mestrado, doutorado) (Farrel, Jimerson, \& Oakland, 2007).

Em Portugal, apesar de a formação dos primeiros psicólogos datar do início da década de 1980, só recentemente foi criada a Ordem dos Psicólogos Portugueses (OPP) (Decreto-Lei n 57/2008). Consequentemente, ainda é incipiente a regulação relativa às áreas de especialidade, razão pela qual qualquer profissional registrado como membro efetivo na OPP poderá exercer funções em meio escolar, independentemente de sua formação teórico-prática. O estudo mais recente, publicamente disponível, com base numa amostra de 318 profis-sionais de SPO, descreveu-os como sendo majoritariamente licenciados em Psicologia (94\%) e especializados em orientação escolar e profissional (36\%), em Psicologia Clínica e consulta psicológica (27\%), ou em Psicologia Educacional (22\%) (CIDEC, 2006).

Sendo a formação uma variável importante, certo é que também não existem guidelines para a formação de psicólogos, verificando-se uma grande diversidade de currículos, modelos teórico-práticos e designações nos cursos de graduação e pós-graduação em Psicologia. A reestruturação do ensino superior em Portugal, em razão da Declaração de Bolonha, não foi acompanhada da definição de um perfil de competências para os psicólogos, tendo tido como principal repercussão a adoção de um modelo de formação em duas etapas sequenciais, constituído por um primeiro ciclo básico em Psicologia, correspondente à licenciatura (três anos), e por um segundo ciclo de especialização, equivalente ao mestrado (dois anos). Assim, se anteriormente à introdução da Declaração de Bolonha uma licenciatura de quatro a cinco anos permitia o acesso à profissão, hoje se exige o mestrado, mantendo-se no entanto os mesmos cinco anos de formação.

A responsabilidade de manter elevados níveis de competência e, consequentemente, de compromisso com a aprendizagem ao longo da vida, é consensualmente reconhecida como uma obrigação ética e profissional (American Psychological Association, 2010; EFPA, 2005; NASP, 2010; OPP, 2008). Se na década de 1980, frente ao rápido desenvolvimento do campo, se estimava que a "meia-vida" para o conhecimento do psicólogo escolar seria de três a cinco anos (Hynd, Pielstick, \& Schakel, 1981), hoje pressupõe-se que essa desatualização ocorra ainda mais rapidamente (Fowler \& Harrisson, 2001). Os benefícios do desenvolvimento profissional continuado encontram-se bem documentados e incluem (a) a atualização e desenvolvimento de novas competências; (b) o desenvolvimento pessoal e social; (c) a capacitação para lidar com a mudança de papéis e funções, problemas emergentes e novas tecnologias; (d) a especialização; e (e) a melhoria da qualidade e eficácia dos serviços prestados (Armistead, 2008; Brown, 2002; Fowler \& Harrison, 1995).

Independentemente desse reconhecimento, um reduzido número de estudos tem procurado analisar de forma compreensiva as necessidades e práticas de desenvolvimento profissional dos psicólogos escolares (Chafouleas, Clonan, \& Vanauken, 2002; Fowler \& Harrison 2001; Lam \& Yuen, 2004). A análise desse tópico tem sido frequentemente realizada no contexto de trabalhos com objetivos amplos, emergindo como um interesse secundário de investigação. No plano empírico, observa-se ainda a propensão para focar as práticas de supervisão, em detrimento de outras formas de desenvolvimento profissional, como a formação continuada (Lam \& Yuen, 2004). Esta última, ainda que menos estudada, tem também chamado a atenção dos pesquisadores (Armistead, Castillo, Curtis, Chappel, \& Cunningham, 2013; Fowler \& Harrison, 2001; Lam \& Yuen, 2004; Wnek, Klein, \& Bracken, 2008).

O enquadramento legal dos SPO prevê o apoio à formação continuada dos psicólogos escolares, mediante a celebração de protocolos entre o Ministério da Educação, instituições do ensino superior e associações científicas e profissionais (Decreto-Lei n 190/91). Essa medida, no entanto, encontra-se longe de ter sido implementada, não se verificando há mais de uma década nenhum planeamento estratégico na área (Sindicato Nacional dos Psicólogos, 2003). Os parcos estudos que têm procurado explorar essas questões apresentam resultados relativamente consistentes, identificando sobretudo necessidades de formação no âmbito das atividades de avaliação e diagnóstico, da intervenção psicope- 
dagógica e psicoterapêutica, da educação especial, do processo ensino-aprendizagem, dos problemas de comportamento e da orientação vocacional (CIDEC, 2006; Ministério da Educação, 2000).

Em Portugal, além de não estar definido um perfil de competências teórico-práticas para os psicólogos escolares, observa-se como agravante a falta de coordenação, supervisão e orientação técnico-científica dos SPO. Nesse contexto, e dada a influência das experiências de formação sobre a qualidade dos serviços profissionais, o presente estudo propôs-se a três objetivos: (a) analisar o perfil de formação dos psicólogos escolares portugueses; (b) identificar suas necessidades de formação; (c) compreender como essas necessidades variam em função de características individuais e dos contextos de trabalho. Procurou-se explorar estas últimas relações, na linha de estudos anteriores (Armistead et al., 2013; Fowler \& Harrison, 2001), uma vez que o perfil de formação, a experiência prévia e as necessidades dos clientes ou contextos de trabalho emergem como variáveis importantes no momento de planejar as atividades de desenvolvimento profissional (Armistead, 2008; Brown, 2002; Fowler \& Harrison, 1995).

Com este artigo pretende-se refletir sobre o perfil de competências desejáveis para o psicólogo escolar, sobre sua formação na graduação e na pós- graduação, bem como sobre a necessidade de formação complementar, visando ao desenvolvimento de competências que atendam às demandas escolares e contemplem o seu desenvolvimento socioprofissional.

\section{Método}

\section{Participantes}

Participaram deste estudo 477 psicólogos escolares, na sua maioria do gênero feminino (88\%) e atuantes no sistema público de ensino (80\%). Em média, reportaram 38 anos de idade (Desvio-Padrão - DP $=8,36$ ) e 12 anos de experiência como psicólogo escolar ( $D P=7,39)$. Trabalhavam em média há 8 anos ( $D P=6,49)$ consecutivos nos estabele- cimentos de ensino em questão, com a seguinte distribuição geográfica: Norte 37\%, Centro 22\%, Sul $29 \%$ e ilhas $12 \%$.

\section{Instrumentos}

Este estudo considerou as respostas dadas em um questionário mais amplo, tomando as questões referentes ao percurso acadêmico e às necessidades de formação dos profissionais da amostra. Os profissionais foram inquiridos acerca do grau acadêmico de que eram detentores, da área de especialização e das áreas em que gostariam de adquirir e/ou aprofundar conhecimentos, de forma a melhorar sua capacidade de resposta frente às exigências do sistema educativo. A formalização desses itens não obedeceu sempre à mesma estrutura, conjugando questões abertas, fechadas e de tipo misto. Dada a dispersão geográfica da população em estudo, este instrumento foi projetado de raiz para uma administração on-line.

\section{Procedimentos}

O recrutamento dos participantes foi realizado via correio eletrônico dirigido aos órgãos de gestão, fornecendo um link e password de acesso ao inquérito e solicitando a participação na pesquisa.

Diante da ausência de uma lista completa e atualizada da população em estudo, bem como da diferente organização do setor público e privado, foram utilizadas estratégias diversificadas na disseminação do inquérito. No ensino público, a divulgação foi assegurada pela Direção Geral da Educação do Ministério da Educação e Ciência, junto a toda a rede escolar, procedendo-se a uma amostragem por saturação. Já os estabelecimentos de ensino privado foram identificados a partir das bases de dados das respetivas Direções e Secretarias Regionais de Educação, assumindo-se essa informação como atualizada. Sempre que o endereço eletrônico da direção se encontrava disponível, essa foi contactada pelo autor principal, procedendo-se a uma amostragem por conveniência. Com vista a aumentar a taxa de resposta, foram implementadas 
outras estratégias de recrutamento e retenção dos participantes, dentre as quais se salientam a utilização de sistemas de lembretes e reforço dos participantes, a divulgação do estudo nas redes sociais (e.g., Facebook, Twitter e fóruns online de profissionais) e junto a pessoas-chave com acesso privilegiado à população. Todo o processo de construção e implementação do inquérito foi executado com recurso ao software LimeSurvey, versão 1.91+, tendo a recolha de dados decorrido entre maio e setembro de 2012. A informação recolhida foi tratada com os programas Statistical Package for the Social Sciences (SPSS) versão 21 e NVivo 10. Análises descritivas foram realizadas e o teste de Qui-quadrado $\left(\chi^{2}\right)$ utilizado para comparações entre grupos. Todo o material resultante de questões abertas foi objeto de análise de conteúdo.

\section{Resultados}

No que concerne ao percurso de formação, $63 \%$ dos psicólogos reportaram como habilitação profissional a licenciatura, 35\% o mestrado e 2\% o doutorado. Apenas uma percentagem residual dos participantes (5\%) mencionou ter realizado um plano de estudos nos moldes da Declaração de Bolonha. A generalidade dos participantes (90\%) referiu algum tipo de especialização desde a graduação, isto é, desde a licenciatura. Dada a diversidade de áreas de especialidade reportadas, tanto na graduação como na pós-graduação, procedeu-se à sua organização em três categorias:

a) Psicologia Escolar e da Educação: com áreas de especialidade aplicadas ao campo da educação e relativas à prática psicológica em contexto escolar, como Psicologia da Educação, Psicologia Escolar, Psicologia do Desenvolvimento da Criança, orientação escolar e vocacional, dentre outras;

b) Psicologia Clínica e da Saúde: referente a áreas de especialidade aplicadas ao campo da saúde e à prática clínica, como Psicologia Clínica, Psicologia da Saúde, aconselhamento e psicoterapia, consulta psicológica de jovens e adultos, dentre outras;

c) Outras áreas de especialidade: agregando grande diversidade de especializações menos frequentes (e.g., Psicologia da justiça, Psicologia do comportamento desviante, Psicologia comunitária, Psicologia do esporte, Psicologia social, Psicologia do trabalho e das organizações, dentre outras).

Assim, entre os psicólogos que reportaram como grau mais elevado a licenciatura com algum tipo de especialização, 53\% referiram ser especializados no domínio da Psicologia Escolar e da Educação, 38\% no âmbito da Psicologia Clínica e da Saúde, e 9\% em outras áreas. Tendência similar foi observada entre os psicólogos com mestrado: 61\% reportaram ser especializados na área da Psicologia Escolar e da Educação, 28\% da Psicologia Clínica e da Saúde, e $11 \%$ em outras áreas. A Tabela 1 permite analisar o perfil de formação de 117 participantes que reportaram áreas de especialização tanto na licenciatura como no mestrado.

Apesar de a maioria dos participantes (60\%) ter mantido no mestrado a mesma área de especialização da licenciatura, uma percentagem substancial dos profissionais (40\%) relatou mudança na especialização. A maioria dos psicólogos que escolheu a área da Psicologia Escolar e da Educação durante a graduação, relatou ter investido nessa mesma área durante o mestrado. Pelo contrário, a maioria dos profissionais provenientes de outras especialidades optaram por áreas não necessariamente relacionadas com a da graduação. Quando considerada a totalidade de áreas de especialidade reportadas na licenciatura, no mestrado e no doutorado, observou-se que $55 \%$ dos participantes referiram algum tipo de especialização na área da Psicologia Escolar e da Educação.

Os psicólogos foram ainda inquiridos acerca de suas necessidades de formação continuada. Essa informação foi recolhida por meio de uma pergunta aberta, que permitia identificar até cinco áreas em que fossem percebidas necessidades de formação. Do total de participantes, cerca de $11 \%$ não identificaram nenhuma necessidade de formação, 36\% identificaram até duas áreas, e 53\% apontaram três ou mais áreas. O número de necessidades de formação identificadas não apresentou uma relação estatisticamente significativa com a variável anos de experiência como psicólogo escolar, $\chi^{2}(6,477)=4.98$, $p=0.54$, nem com o fato de os psicólogos pos- 
suírem ou não algum tipo de especialização na área da Psicologia Escolar e da Educação, $\chi^{2}(2,477)$ = 3.97, $p=0.14$.

A Tabela 2 sumariza as necessidades de formação continuada dos participantes em estudo. Para proceder a uma melhor sistematização da informação recolhida, as respostas foram organizadas em categorias mais abrangentes.

As necessidades de formação referidas apontam grande diversidade de problemáticas e áreas de intervenção. Dentre os problemas mais frequentemente identificados encontram-se as dificuldades de aprendizagem (13\%), a avaliação psicológica (12\%), a educação especial (11\%), a consulta psicológica (10\%) e a indisciplina e problemas de comportamento (9\%). Apesar de na maioria dos casos essa identificação ter sido feita de forma generalista, alguns participantes foram mais pormenorizados quanto à natureza de suas necessidades. Por exemplo, no caso da avaliação psico-

Tabela 1

Perfil de formação de acordo com a área de especialização $(n=117)$

\begin{tabular}{|c|c|c|}
\hline \multicolumn{3}{|c|}{ Área de especialização } \\
\hline Licenciatura & Mestrado & $\%$ \\
\hline \multirow[t]{3}{*}{ Psicologia Escolar/Educação } & Psicologia Escolar e da Educação & 41.0 \\
\hline & Psicologia Clínica e da Saúde & 8.5 \\
\hline & Outras áreas de especialidade & 2.6 \\
\hline \multirow[t]{3}{*}{ Psicologia Clínica/Saúde } & Psicologia Clínica e da Saúde & 18.8 \\
\hline & Psicologia Escolar e da Educação & 18.8 \\
\hline & Outras áreas de especialidade & 1.7 \\
\hline \multirow[t]{2}{*}{ Outras áreas } & Psicologia Escolar e da Educação & 6.0 \\
\hline & Psicologia Clínica e da Saúde & 2.6 \\
\hline
\end{tabular}

Tabela 2

Áreas e conteúdos em que foram identificadas necessidades de formação

\begin{tabular}{|c|c|c|}
\hline Categorias & $\mathrm{n}$ & $\%$ \\
\hline Dificuldades de aprendizagem: remete para avaliação e intervenção nas várias dificuldades de aprendizagem. & 159 & 13 \\
\hline $\begin{array}{l}\text { Avaliação: refere à avaliação psicológica, educacional e neuropsicológica no sentido lato, ao uso de testes e elaboração de } \\
\text { relatórios. }\end{array}$ & 146 & 12 \\
\hline Educação especial: reúne alusões gerais à educação especial e a um conjunto de atividades circunscritas a essa área. & 136 & 11 \\
\hline Consulta psicológica: abrange o aconselhamento, consulta e psicoterapia, nas suas diferentes vertentes. & 119 & 10 \\
\hline Indisciplina e problemas de comportamento: engloba a avaliação e intervenção quanto a dificudades de comportamento. & 116 & 9 \\
\hline $\begin{array}{l}\text { Educação parental e intervenção familiar: compreende a formação, o aconselhamento parental, a intervenção sistêmica e } \\
\text { familiar. }\end{array}$ & 96 & 8 \\
\hline Promoção de competências: abarca intervenções que visam o aumento de competências socioafetivas cognitivas e acadêmicas. & 95 & 8 \\
\hline Orientação vocacional: contém referências gerais à orientação vocacional, a instrumentos e a intervenções nessa área. & 93 & 8 \\
\hline Educação para a saúde: inclui a promoção de comportamentos de saúde e prevenção de comportamentos de risco. & 52 & 4 \\
\hline Desenvolvimento normativo e não normativo: refere-se à psicologia e psicopatologia do desenvolvimento. & 44 & 4 \\
\hline Gestão de conflitos e mediação escolar: inclui intervenções que visam à resolução positiva de conflitos no meio escolar. & 42 & 3 \\
\hline Consultoria: reúne referências gerais à prática de consultoria, ou especificamente à consultoria a professores e ao sistema. & 40 & 3 \\
\hline Legislação e ética: remete para legislação relevante à prática profissional, à ética e à deontologia. & 23 & 2 \\
\hline Violência escolar: concerne à avaliação, prevenção e intervenção nas diferentes formas de violência escolar. & 18 & 1 \\
\hline Metodologias de ensino e pedagogia diferenciada: diz respeito aos métodos de ensino, à diferenciação pedagógica e curricular. & 17 & 1 \\
\hline $\begin{array}{l}\text { Outros: agrega tópicos de natureza diversificada, de frequência residual (por exemplo, maus-tratos, métodos e técnicas de } \\
\text { investigação, metodologia de projeto, dentre outras). }\end{array}$ & 44 & 4 \\
\hline
\end{tabular}


lógica, foi encontrada a expressão de objetivos de "atualização", "reciclagem" e "contato com novos instrumentos". No âmbito da educação especial, foram detalhadas necessidades de formação nas áreas de avaliação e intervenção nas demandas educativas especiais, elaboração de documentos nesse domínio e, ainda, quanto à Classificação Internacional de Funcionalidade, Incapacidade e Saúde. Já no nível da consulta psicológica, observou-se um interesse particular pelas abordagens breves, cognitivo-comportamentais e modalidades de intervenção em grupo. A expressão "programas" foi encontrada frequentemente associada às categorias "promoção de competências e educação para a saúde" sugerindo que, mais do que obter formação de natureza geral nessas áreas, os profissionais desejam aceder a intervenções estruturadas, que rapidamente possam ser transferidas para seus contextos de trabalho.

Pressupondo que as necessidades de formação dos psicólogos escolares possam variar de acordo com as características dos profissionais e do contexto de trabalho, procedeu-se à sua análise em função de variáveis como (a) anos de experiência como psicólogo escolar (Tabela 3); (b) formação especializada, ou não, na área da Psicologia Escolar e da Educação (Tabela 4); e (c) tipologia do estabelecimento de ensino (Tabela 5).
A leitura das Tabelas 3, 4 e 5 permite constatar a ausência de um padrão consistente de variabilidade quanto às necessidades de formação reportadas em função daquelas três variáveis. Observaram-se, no entanto, algumas tendências merecedoras de atenção, as quais deverão ser interpretadas com prudência, dado o reduzido número de respostas em algumas categorias e grupos analisados. Nesse sentido, destacam-se os seguintes aspectos: (a) os psicólogos escolares mais experientes (isto é, $\geq 16$ anos) identificaram com mais frequência as necessidades de formação nas áreas de avaliação, consulta psicológica e consultoria; (b) os psicólogos com especialização em educação mencionaram com mais frequência as necessidades de formação no âmbito da consulta psicológica, do desenvolvimento normativo e não normativo; (c) os psicólogos escolares que trabalhavam em estabelecimentos de ensino que incluíam apenas o nível básico (isto é, os primeiros nove anos de escolaridade) referiram com mais frequência as necessidades de formação em dificuldades de aprendizagem, indisciplina/problemas de comportamento, violência escolar, educação familiar e intervenção parental, (d) os profissionais de escolas que incluíam apenas o ensino secundário reportaram mais frequentemente necessidades de formação em orientação vocacional.

Tabela 3

Distribuição das categorias em função do número de anos de experiência como psicólogo escolar

\begin{tabular}{|c|c|c|c|c|c|c|c|c|}
\hline \multirow{3}{*}{ Categorias } & \multicolumn{8}{|c|}{ Anos de experiência como psicólogo escolar } \\
\hline & \multicolumn{2}{|c|}{$\leq 5(n=112)$} & \multicolumn{2}{|c|}{$6-10(n=121)$} & \multicolumn{2}{|c|}{$11-15(n=100)$} & \multicolumn{2}{|c|}{$\geq 16(n=144)$} \\
\hline & $\mathrm{n}$ & $\%$ & $\mathrm{n}$ & $\%$ & $\mathrm{n}$ & $\%$ & $n$ & $\%$ \\
\hline Dificuldades de aprendizagem & 39 & 35 & 42 & 35 & 35 & 35 & 43 & 30 \\
\hline Avaliação & 24 & 21 & 36 & 30 & 24 & 24 & 62 & 43 \\
\hline Educação especial & 39 & 35 & 39 & 32 & 32 & 32 & 26 & 18 \\
\hline Consulta psicológica & 22 & 20 & 24 & 20 & 15 & 15 & 58 & 40 \\
\hline Indisciplina e problemas de comportamento & 22 & 20 & 32 & 26 & 20 & 20 & 42 & 29 \\
\hline Educação parental e intervenção familiar & 24 & 21 & 23 & 19 & 29 & 29 & 20 & 14 \\
\hline Promoção de competências & 18 & 16 & 27 & 22 & 16 & 16 & 34 & 24 \\
\hline Orientação vocacional & 22 & 20 & 20 & 17 & 17 & 17 & 34 & 24 \\
\hline Educação para a saúde & 15 & 13 & 17 & 14 & 9 & 9 & 11 & 8 \\
\hline Desenvolvimento normativo e não normativo & 13 & 12 & 11 & 9 & 10 & 10 & 10 & 7 \\
\hline Consultoria & 5 & 4 & 9 & 7 & 2 & 2 & 24 & 17 \\
\hline
\end{tabular}


Tabela 4

Distribuição das categorias em função de possuir ou não formação especializada em Psicologia Escolar e da Educação

\begin{tabular}{|c|c|c|c|c|}
\hline \multirow{3}{*}{ Categorias } & \multicolumn{4}{|c|}{ Formação em Psicologia Escolar e da Educação } \\
\hline & \multicolumn{2}{|c|}{$\operatorname{Sim}(n=263)$} & \multicolumn{2}{|c|}{ Não $(n=214)$} \\
\hline & $\mathrm{n}$ & $\%$ & $\mathrm{n}$ & $\%$ \\
\hline Dificuldades de aprendizagem & 84 & 32 & 75 & 35 \\
\hline Avaliação & 88 & 33 & 58 & 27 \\
\hline Educação especial & 74 & 28 & 62 & 29 \\
\hline Consulta psicológica & 78 & 30 & 41 & 19 \\
\hline Indisciplina e problemas de comportamento & 68 & 26 & 48 & 22 \\
\hline Educação parental e intervenção familiar & 54 & 21 & 42 & 20 \\
\hline Promoção de competências & 52 & 20 & 43 & 20 \\
\hline Orientação vocacional & 44 & 17 & 49 & 23 \\
\hline Educação para a saúde & 27 & 10 & 25 & 12 \\
\hline Desenvolvimento normativo e não normativo & 32 & 12 & 12 & 6 \\
\hline Gestão de conflitos e mediação escolar & 25 & 10 & 17 & 8 \\
\hline Consultoria & 22 & 8 & 18 & 8 \\
\hline
\end{tabular}

Tabela 5

Distribuição das categorias em função da tipologia do estabelecimento de ensino

\begin{tabular}{|c|c|c|c|c|}
\hline \multirow{3}{*}{ Categorias } & \multicolumn{4}{|c|}{ Tipologia do estabelecimento de ensino } \\
\hline & \multicolumn{2}{|c|}{ Básico ( $n=217)$} & \multicolumn{2}{|c|}{ Secundário $(n=116)$} \\
\hline & $\mathrm{n}$ & $\%$ & $\mathrm{n}$ & $\%$ \\
\hline Dificuldades de aprendizagem & 77 & 35 & 27 & 23 \\
\hline Avaliação & 62 & 29 & 41 & 35 \\
\hline Educação especial & 68 & 31 & 33 & 28 \\
\hline Consulta psicológica & 49 & 23 & 28 & 24 \\
\hline Indisciplina e problemas de comportamento & 54 & 25 & 22 & 19 \\
\hline Educação parental e intervenção familiar & 55 & 25 & 22 & 19 \\
\hline Promoção de competências & 42 & 19 & 18 & 16 \\
\hline Orientação vocacional & 30 & 14 & 31 & 27 \\
\hline Educação para a saúde & 20 & 9 & 15 & 13 \\
\hline Desenvolvimento normativo e não normativo & 25 & 12 & 8 & 7 \\
\hline Gestão de conflitos e mediação escolar & 18 & 8 & 12 & 10 \\
\hline Consultoria & 13 & 6 & 12 & 10 \\
\hline
\end{tabular}

\section{Discussão}

Procurou-se neste estudo analisar o perfil de formação dos psicólogos escolares, bem como aferir suas necessidades de formação. Quanto à graduação, observou-se que a maioria dos psicólogos escolares concluíra a licenciatura antes da implementação da Declaração de Bolonha e se especializara em áreas aplicadas à educação. Menos frequente, mas em número relevante, são os profissionais detentores de mestrado e provenientes 412 de áreas como a Psicologia Clínica e da Saúde.
Prevê-se, no entanto, a médio e longo prazo, um aumento de profissionais com mestrado e especializado no campo da educação, decorrente da reorganização do ensino superior à luz da Declaração Bolonha, e da futura criação de um quadro de especialidades em Psicologia.

Tanto o mestrado como a formação especializada são geralmente requeridos, e por isso mais frequentes, nos países desenvolvidos e com normas mais exigentes para o exercício da profissão (Farrell et al., 2007). Por outro lado, o grau de qualificação dos psicólogos escolares tende a refletir as caracte- 
rísticas do sistema educativo e as opções de formação disponíveis em cada país (Jimerson, Graydon, Curtis, \& Staskal, 2007). A análise dessas condições em Portugal permite compreender o predomínio do grau de licenciado entre os psicólogos escolares, sendo esta também a situação em vários outros países (Guzzo, Martínez, \& Campos, 2007; Jimerson et al., 2007; Jimerson et al., 2008) com uma graduação em Psicologia de cinco anos, e não uma formação em duas etapas e conducente ao mestrado, como acontece hoje nos países europeus que aderiram à Declaração de Bolonha.

Dada a importância de envolver os profissionais na sinalização das áreas em que necessitam se atualizar, aperfeiçoar ou expandir competências, os psicólogos escolares foram inquiridos acerca dessa temática. Os resultados obtidos apontam uma grande diversidade de necessidades de formação, reflexo da natureza multifacetada do papel do psicólogo escolar, da multiplicidade de problemáticas, e populações que podem beneficiar da ação dos SPO. Relativamente aos seus antecessores (CIDEC, 2006; Ministério da Educação, 2000), este estudo identifica um leque mais vasto de necessidades de formação, observando-se todavia consistência nas áreas priorizadas pelos profissionais.

Dentre as necessidades de formação mais enumeradas, destacam-se: avaliação psicológica, consulta psicológica, educação especial, orientação vocacional, e dificuldades de aprendizagem e comportamento. Esses resultados espelham as áreas de atuação tradicional dos psicólogos escolares, em torno das quais tende a se concentrar a ação dos SPO. Dados recentes sugerem que a avaliação, a consulta psicológica, a orientação vocacional e a educação especial ocupam em média $65 \%$ do tempo de trabalho dos psicólogos escolares portugueses, e que essas são geralmente as atividades mais solicitadas pela comunidade (Mendes, Abreu-Lima, \& Almeida, 2013b; Mendes et al., 2014). Esses resultados não são surpreendentes quando se considera que os psicólogos escolares são (a) comumente associados à determinação da elegibilidade para a educação especial e às tarefas de avaliação em geral (Fagan \& Wise, 2007; Farrel, 2010; Riccio, Garcia-Vasques, \& Crespi, 2010); (b) soli- citados para atuar junto a alunos com algum tipo de dificuldade acadêmica, comportamental ou emocional (Almeida, 2003; Almeida \& Peixoto, 2011; Lowell, Rimmer, \& Zeeman, 2010), e (c) percebidos pelo sistema escolar como um profissional cuja atuação se foca no indivíduo, na identificação e resolução de problemas (Pasqualini, Souza, \& Lima, 2013; Souza, Ribeiro, \& Silva, 2011). Parece plausível que os profissionais desejem alcançar elevados níveis de proficiência nas áreas em que primordialmente intervêm ou são chamados a intervir.

Apesar de menos frequente, um número substancial de participantes evidenciou o interesse em adquirir ou aprofundar conhecimentos na área da educação parental, da intervenção familiar e do desenvolvimento de competências de crianças e jovens. Esses resultados sugerem o reconhecimento, por parte de alguns profissionais, da necessidade de uma intervenção mais contextual, voltada para o desenvolvimento integral do aluno e sua família. Apesar dessa visão mais ecológica, não se pode deixar de sublinhar o reduzido número de psicólogos escolares que identificou necessidades de formação voltada para a intervenção junto de professores e outros profissionais, ou voltada à instituição escolar no seu todo. Entre as oportunidades de atuação nesse âmbito, estão a formação, a consultoria e supervisão de outros técnicos, a participação na elaboração do projeto educativo da escola, a atuação nos órgãos de gestão escolar e organização pedagógica, a implementação de projetos nas escolas e, ainda, a articulação com outros serviços e instituições da comunidade. Trata-se de uma linha de intervenção mais indireta, considerada mais promocional e eficaz, na direção da qual os serviços têm sido fortemente incentivados a trabalhar (Almeida, 2003; Almeida et al., 2013; Almeida \& Peixoto, 2011; Farrel, 2010; Gutkin, 2012; Guzzo et al., 2001).

Resta comentar esses resultados à luz da investigação internacional. Um ponto comum entre os resultados deste estudo e os internacionais invocados é o fato de que a prestação de serviços diretos, dirigida aos alunos com problemas socioemocionais, comportamentais ou de aprendizagem, emerge como área prioritária para formação. No que diz 
respeito à intervenção indireta, as tendências observadas não são tão claras. Se em alguns estudos ela aparece como uma das áreas em que os psicólogos escolares identificam mais necessidades de formação (Fowler \& Harrison, 2001; Lam \& Yuen, 2004), em outros ela é uma área nitidamente secundária, tal como sucede nesta investigação (Armistead et al., 2013; Wnek et al., 2008). Também nesses trabalhos não tem sido possível observar uma relação significativa entre necessidades de formação e variáveis individuais e contextuais (Armistead et al., 2013; Fowler \& Harrison, 2001).

Apesar de apresentar informações relevantes, este estudo não é isento de limitações. As principais delas advêm: (a) do uso de técnicas de amostragem não probabilísticas, o que coloca ressalvas à generalização dos resultados; (b) da dificuldade de assegurar a divulgação do inquérito a todos os psicólogos escolares, existindo o risco de diferenças entre respondentes e não respondentes; e (c) do uso de um instrumento de autorrelato e, por isso, baseado na perceção dos próprios respondentes. $\mathrm{A}$ restrição da resposta à enumeração de até cinco necessidades constitui também uma limitação, sendo legítimo questionar se diferentes resultados teriam sido obtidos com uma questão livre.

A partir deste estudo, podem ser derivadas implicações tanto para a formação inicial quanto para a formação continuada dos psicólogos escolares. Antes de tudo, os resultados encontrados refletem a complexidade e a especificidade do perfil de formação desses profissionais. Dos psicólogos escolares é esperada a capacidade de desempenhar uma multiplicidade de funções, atuar junto a diferentes públicos-alvo, responder a uma magnitude de problemas e implementar uma gama de serviços que vão da prevenção à remediação, da intervenção direta à indireta. A graduação encontra aqui a responsabilidade de dotar os profissionais de um conjunto de competências teórico-práticas que Ihes permita corresponder a essas expectativas. A concretização desse objetivo implica clareza acerca dos valores, princípios e quadros conceituais que devem orientar a ação dos psicólogos escolares, razão pela qual é importante avançar na definição do perfil de competências desses profissionais em Portugal.
Paralelamente, e atendendo à diversidade da formação de base dos psicólogos escolares portugueses, é urgente implementar políticas públicas de apoio à formação continuada desses profissionais. Essas atividades só farão sentido se se aproximarem da práxis dos psicólogos escolares, promovendo a reflexão em serviço (Marinho-Araújo $\&$ Neves, 2007). Através da formação continuada gera-se o desafio, mas também a oportunidade, de promover nos psicólogos escolares o desenvolvimento de competências que Ihes permitam responder às necessidades das escolas, sem que fiquem confinados ao modelo clínico de intervenção escolar, isto é, a uma prática centrada nas atividades de diagnóstico, tratamento e remediação.

Em Portugal, a Psicologia Escolar é ainda uma área em desenvolvimento e afirmação. Presentemente, não está definido um perfil de competências para o exercício profissional da Psicologia em contexto escolar, assim como também não existem diretrizes para a formação nessa especialidade. Nem mesmo a denominação psicólogos escolares é consensual. Questões relativas ao perfil de formação desses profissionais ganham particular relevância frente à ausência de guidelines, coordenação e supervisão dos serviços. Acreditando que nos próximos anos essas lacunas serão progressivamente preenchidas, não se pode deixar de sublinhar a importância de, por um lado, garantir que os profissionais da área estejam capacitados para responder às necessidades e desafios dos contextos escolares contemporâneos e, por outro, assegurar a preparação da futura geração de psicólogos escolares, em áreas básicas e aplicadas, nos domínios da Psicologia e da Educação.

Por último, dado que este trabalho não pretendeu esgotar a análise e discussão dessas questões, novos estudos são necessários. Entre os tópicos propostos para investigação estão a análise curricular dos programas de formação em Psicologia Escolar e Psicologia da Educação, explorando disciplinas, áreas de enfoque e referenciais teóricos subjacentes à formação. Igualmente importante é o estudo das práticas de formação continuada dos psicólogos escolares, examinando os tópicos, modalidades e durabilidade das atividades frequentadas, 
a periodicidade do envolvimento nesses tipos de ação, as motivações implícitas e as principais barreiras à sua frequência. Futuras pesquisas poderão ainda procurar refinar os resultados obtidos neste estudo, por meio de questões fechadas e de entrevistas a subgrupos de psicólogos escolares, organizados por caraterísticas específicas.

\section{Referências}

Almeida, L. S. (2003). Psicologia escolar em Portugal. In S. N. Neves (Ed.), Psicologia em Portugal (pp.13-44). Coimbra: Quarteto Editora.

Almeida, L. S., Franco, A., Soares, L. D., Alves, A. F., \& Gonçalves, P. (2013). O psicólogo escolar face aos desafios da escola de futuro. In I. E. Rego \& S. N. Caldeira (Eds.), Prevenir ou remediar: contextos para a intervenção em psicologia (pp.17-41). Braga: Psiquilibrios.

Almeida, L. S., \& Peixoto, F. (2011). Psicologia da educação. In M. P. Lopes, P. J. Palma, R. B. Ribeiro, \& M. Pina e Cunha (Eds.), Psicologia aplicada (pp.47-68). Lisboa: $\mathrm{RH}$.

American Psychological Association. (2010). Ethical principles of psychologists and code of conduct. Retrieved November 24, 2013, from http://www.apa. org/ethics/code/principles. pdf

Armistead, L. D. (2008). Best practices in continuing professional development for school psychologists. In A. Thomas \& J. Grimes (Eds.), Best practices in school psychology V (pp.1975-1989). Bethesda, MD: National Association of School Psychologists.

Armistead, L. D., Castillo, J. M., Curtis, M. J., Chappel, A., \& Cunningham, J. (2013). School psychologists' continuing professional development preferences and practices. Psychology in the Schools, 50(4), 415-432. http://dx.doi.org/10.1002/pits.21684

Barbosa, R. M., \& Marinho-Araújo, C. M. (2010). Psicologia escolar no Brasil: considerações e reflexões históricas. Estudos de Psicologia (Campinas), 27(3), 393-402. http://dx.doi.org/10.1590/S0103-166X2010000 300011

Brown, M. (2002). Best practices in professional development. In A. Thomas \& J. Grimes (Eds.), Best practices in school psychology IV (pp.183-194). Bethesda, MD: National Association of School Psychologists.

Centro Interdisciplinar de Estudos Económicos. (2006). Estudo sobre a intervenção no contexto escolar dos serviços de psicologia e orientação. Lisboa: Direção Geral de Inovação e Desenvolvimento Curricular.

Chafouleas, S. M., Clonan, S. M., \& Vanauken, T. L. (2002). A national survey of current supervision practices and evaluation practices of school psychologists. Psychology in the Schools, 39(3), 317-325.

Decreto-Lei no 190/91. (Portugal, 1991, 17 de maio). Cria nos estabelecimentos de educação e ensino públicos os serviços de psicologia e orientação. Recuperado em novembro 24, 2013, de http://dre.pt/pdf1sdip/ 1991/05/113a00/26652669.PDF

Decreto-Lei n 57/2008. (Portugal, 2008, 4 de setembro). Cria a Ordem dos Psicólogos Portugueses e aprova o seu estatuto. Recuperado em novembro 24, 2013, de http://www.ordemdospsicologos.pt/ficheiros/ documentos/lei_57_2008_de_4_de_setembro.pdf

European Federation of Psychologists' Associations. (2005). Principles for professional ethics. Retrieved November 24, 2013, from http://www.efpa.eu/ethics

European Federation of Psychologists' Associations. (2010). EFPA position paper on psychologists in the educational system and their contribution to lifelong learning - October 2010. Retrieved November 24, 2013, from http://www.efpa.eu/download/72647 dc67149b92876bcc25cf46ebd07

Fagan, T. K., \& Wise, P. S. (2007). School Psychology: Past, present and future ( $3^{\text {rd }}$ ed.). Bethesda, MD: National Association of School Psychologists.

Farrell, P. (2010). School psychology: Learning lessons from history and moving forward. School Psychology International, 31(6), 581-598. http//:www.dx.doi.org/ $10.1177 / 0143034310386533$

Farrell, P., Jimerson, S., \& Oakland, T. (2007). School psychology internationally: A synthesis of findings. In S. Jimerson, T. Oakland, \& P. Farrell (Eds.), The handbook of international school psychology (pp.501-510). Thousand Oaks, CA: Sage.

Fowler, E., \& Harrison, P. L. (1995). Best practices in continuing professional development for school psychologists. In A. Thomas \& J. Grimes (Eds.), Best practices in school psychology III (pp.81-89). Bethesda, MD: National Association of School Psychologists.

Fowler, E., \& Harrison, P. L. (2001). Continuing professional development needs and activities of school psychologists. Psychology in the Schools, 38(1), 75-88.

Gutkin, T. B. (2012). Ecological psychology: Replacing the medical model paradigm for school-based psychological and psychoeducational services. Journal of Educational and Psychological Consultation, 22(1-2), 1-20. http://dx. doi.org/10.1080/10474412.2011.6 49652

Guzzo, R. S. L., Almeida, L. S., \& Wechsler, S. M. (2001). Psicologia escolar: padrões e práticas em países de língua espanhola e portuguesa ( $2^{\mathrm{a}}$ ed.). Campinas: Alínea.

Guzzo, R. S. L., Martínez, A., \& Campos, H. (2007). School psychology in Brazil. In S. Jimerson, T. Oakland, \& P. Farrell (Eds.), The handbook of international school psychology (pp.29-39). Thousand Oaks, CA: Sage. 
Hynd, G. W., Pielstick, N. L., \& Schakel, J. A. (1981). Continuing professional development in school psychology: Current status. School Psychology Review, 10(4), 480-486.

Jimerson, S. R., Graydon, K., Curtis, M. J., \& Staskal, R. (2007). The international school psychology survey: Insights from school psychologists around the world. In S. R. Jimerson, T. D. Oakland, \& P. T. Farrell (Eds.), The handbook of international school psychology (pp.453-463). London: Sage.

Jimerson, S. R., Graydon, K., Skokut, M., Alghorani, M. A., Kanjaradze, A., Forster, J., \& the ISPA Research Committee. (2008). The international school psychology survey: Data from Georgia, Switzerland and the United Arab Emirates. School Psychology International, 29(1), 5-28. http://dx.doi.org/10.1177/ 0143034307088501

Jimerson, S. R., Skokut, M., Cardenas, S., Malone, H., \& Stewart, K. (2008). Where in the world is school psychology? Examining evidence of school psychology around the globe. School Psychology International, 29(2), 131-144.

Lam, S., \& Yuen, M. (2004). Continuing professional development in school psychology. School Psychology International, 25(4), 480-494.

Leitão, L. M., Paixão, M. P., Silva, J. T., \& Miguel, J. P. (2001). Ser psicólogo em contexto escolar: os Serviços de Psicologia e Orientação (SPO's) na região centro. Psychologica, 26, 27-53.

Lowell, C. N., Rimmer, R. J., \& Zeeman, R. D. (2010). Preparing students for leadership roles. In J. Kaufman, T. Hughes, \& C. Riccio (Eds.), Handbook of education, training, and supervision of school psychologists in school and community (Vol.1, pp.149-164). New York: Taylor Francis/Routledge.

Marinho-Araújo, C. M. (2005a). Psicologia escolar e o desenvolvimento de competências. Boletim Academia Paulista de Psicologia, 25(2), 73-85.

Marinho-Araújo, C. M. (2005b). Psicologia escolar: fios e desafios constitutivos de identidade, formação e atuação. Boletim Academia Paulista de Psicologia, 25(2), 88-98.

Marinho-Araújo, C. M., \& Neves, M. M. B. J. (2007). Psicologia escolar e a formação continuada em serviço: encurtando distâncias entre teorias e práticas. Boletim Academia Paulista de Psicologia, 27(1), 56-71.

Mendes, S. A., Abreu-Lima, I., \& Almeida, L. S. (2013a). Psicólogos escolares em Portugal: contributo para a sua caracterização. Psicologia, Educação e Cultura, 17(1),190-208.

Mendes, S. A., Abreu-Lima, I., \& Almeida, L. S. (2013b). A psicologia e orientação em contexto escolar: resultados de um inquérito nacional. In P. Cunha \& F.
Pereira (Chair), Seminário de Psicologia e Orientação em Contexto Escolar. Lisboa, Portugal.

Mendes, S., Abreu-Lima, I., Almeida, L. S., \& Simeonsson, R. J. (2014). School psychology in Portugal: Practitioners' characteristics and practices. International Journal of School \& Educational Psychology, 2(2), 115-125. http:// dx.doi.org/10.1080/21683603.2013.863171

Ministério da Educação. (Portugal, 2000). Serviços de psicologia e orientação: observatório dos serviços de psicologia e orientação. Lisboa: DEB/NOEE.

National Association of School Psychologists. (2010). Model for comprehensive and integrated school psychological services. School Psychology Review, 39(2), 320-333.

Oakland, T. D., \& Cunningham, J. L. (1992). A survey of school psychology in developed and developing countries. School Psychology International, 13(2), 99-129.

Oakland, T. D., \& Jimerson, S. R. (2007). School psychology internationally: A retrospective view and influential conditions. In S. R. Jimerson, T. D. Oakland, \& P. T. Farrell (Eds.), The handbook of international school psychology (pp.453-462). Thousand Oaks, CA: Sage.

Ordem dos Psicólogos Portugueses. (2008). Código deontológico da ordem dos psicólogos. Recuperado em novembro 24, 2013, de https://www.ordemdos psicologos.pt/ficheiros/documentos/codigo_de ontologico.pdf

Pasqualini, M. G., Souza, M. P. R., \& Lima, C. P. (2013). Atuação do psicólogo escolar na perspetiva de proposições legislativas. Psicologia Escolar e Educacional, 17(1), 15-24.

Riccio, C. A., Garcia-Vasques, E., \& Crespi, T. D. (2010). School psychology as a profession: Introduction and overview. In J. Kaufman, T. Hughes, \& C. Riccio (Eds.), Handbook of education, training, and supervision of school psychologists in school and community (Vol.1, pp.3-13). New York: Taylor Francis/Routledge.

Sindicato Nacional dos Psicólogos. (2003). Os psicólogos na educação: que presente e futuro? Recuperado em novembro 24, 2013, de http://www. snp.pt/osindicato/ documentos_files/030717psieduc.htm

Souza, C. S., Ribeiro, M. J., \& Silva, S. M. C. (2011). A atuação do psicólogo escolar na rede particular de ensino. Psicologia Escolar e Educacional, 15(1), 53-61.

Wnek, A. C., Klein, G., \& Bracken, B. A. (2008). Professional development issues for school psychologists: What's hot, what's not in the United States. School Psychology International, 29(2), 145-160. http://dx.doi.org/10.11 $77 / 0143034308090057$

Recebido: agosto 22, 2013

Versão final: dezembro 11, 2013

Aprovado: fevereiro 4, 2014 